\title{
Spin-orbit alignment of exoplanet systems: analysis of an ensemble of asteroseismic observations
}

\author{
Tiago L. Campante ${ }^{1,2}$ \\ ${ }^{1}$ School of Physics and Astronomy, University of Birmingham, \\ Edgbaston, Birmingham, B15 2TT, UK \\ email: campante@bison.ph.bham.ac.uk \\ ${ }^{2}$ Stellar Astrophysics Centre (SAC), Department of Physics and Astronomy, \\ Aarhus University, Ny Munkegade 120, DK-8000 Aarhus C, Denmark
}

\begin{abstract}
Measuring the obliquities of exoplanet-host stars provides invaluable diagnostic information for theories of planetary formation and migration. Most of these results have so far been obtained by measuring the Rossiter-McLaughlin effect, clearly favoring systems that harbor hot Jupiters. While it would be extremely helpful to extend these measurements to long-period and multiple-planet systems, it is also true that the latter systems tend to involve smaller planets, making it ever so difficult to apply such techniques. Asteroseismology provides a powerful method of determining the inclination of the stellar spin axis - from an analysis of the rotationallyinduced splittings of the oscillation modes - whose applicability is ultimately determined by the stellar parameters and not by the signal-to-noise ratio of the transit data. Here we present the first statistical analysis of an ensemble of asteroseismic obliquity measurements obtained for solar-type stars with transiting planets. The sample consists of $25 \mathrm{Kepler}$ planet-candidate host stars, 14 of which are multi-transiting systems. We seek empirical constraints on the spin-orbit alignment of exoplanet systems and discuss the implications for theories of planetary formation and migration.
\end{abstract}

Keywords. asteroseismology, methods: statistical, planetary systems, planets and satellites: general, stars: solar-type, techniques: photometric

\section{Introduction}

For practical reasons, most obliquity measurements to date have been for systems harboring hot Jupiters. Empirical evidence has been found that the obliquities of hotJupiter systems are affected by tidal evolution (e.g., Albrecht et al. 2012): systems with strong planet-star tidal interactions tend to have low obliquities, while systems with weaker tides display a broad range of obliquities that, besides well-aligned planets, also include planets in polar or even retrograde orbits. This suggests that the orbital plane has changed relative to the plane of the protoplanetary disk by the time hot Jupiters are formed and before tides had any effect on the system, which presumably happened due to the same mechanism responsible for their migration.

However, in order to study the dynamical histories of planetary systems across a wider range of architectures, it is imperative to extend these measurements to systems with smaller planets, longer-period planets, and multiple planets. An alternative technique for measuring the obliquities of planetary systems, one that does not depend on the signalto-noise ratio of the transit data, is asteroseismology. The asteroseismic estimation of the stellar inclination angle rests on our ability to resolve and extract signatures of rotation in the power spectra of non-radial modes of oscillation (e.g., Gizon \& Solanki 2003). 


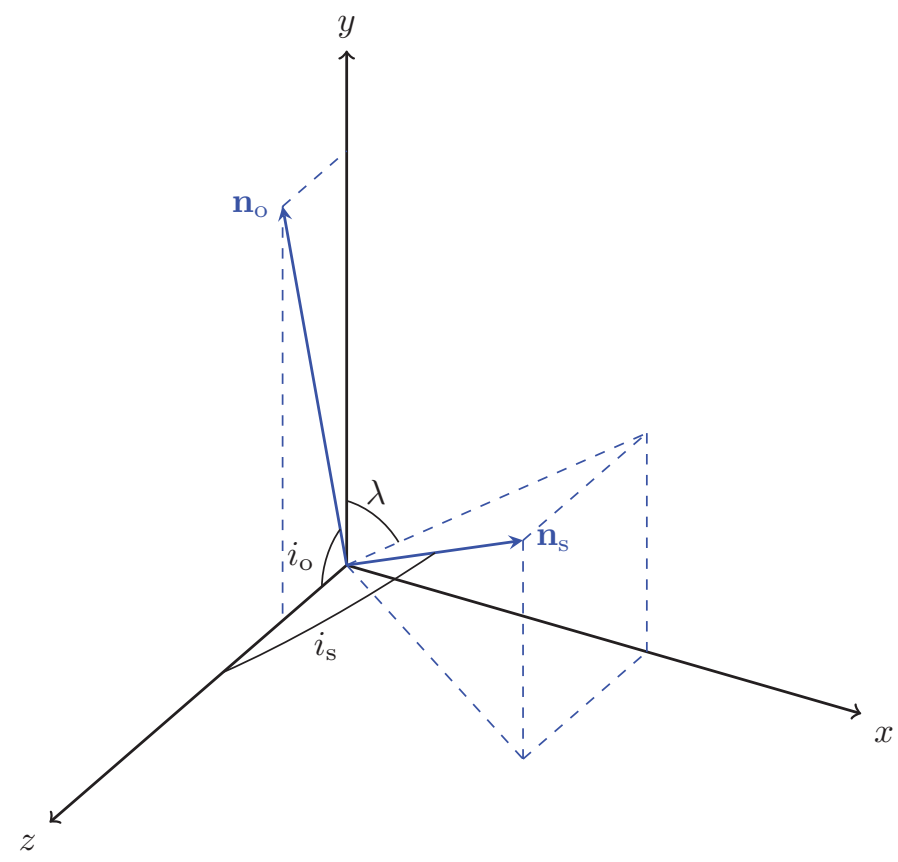

Figure 1. Observer-oriented coordinate system. Here the $z$ axis points toward the observer, the $x$ axis points along the line of nodes, the $y$ axis completes a right-handed triad, and the $x y$ plane is the plane of the sky. The unit vectors $\mathbf{n}_{\mathrm{o}}$ and $\mathbf{n}_{\mathrm{s}}$ denote the orbital and stellar angular momentum unit vectors, respectively. All depicted angles are introduced in the text.

Its applicability depends entirely on the stellar properties and not on the planetary or orbital parameters, which is a clear advantage when measuring the obliquities of systems with small planets or long-period planets. Following its application to a couple of host stars with single, non-transiting large planets discovered using the radial-velocity method (Wright et al. 2011; Gizon et al. 2013), the asteroseismic technique has been recently applied to several solar-type hosts observed with Kepler (e.g., Chaplin et al. 2013; Lund et al. 2014). But perhaps the most significant application to date has been the measurement of a large obliquity for Kepler-56 (Huber et al. 2013), a red giant hosting two transiting planets. This showed that spin-orbit misalignments are not confined to hot-Jupiter systems.

\section{Spin-orbit geometry}

We briefly introduce the geometry of the spin-orbit problem. In Fig. 1, $z$ points toward the observer and the $x y$ plane is the plane of the sky. Moreover, $\mathbf{n}_{\mathrm{o}}$ and $\mathbf{n}_{\mathrm{s}}$ denote the orbital and stellar angular momentum vectors, respectively. For a transiting planet, the orbital inclination $i_{\mathrm{o}}$ is measurable via transit photometry, while the projected spinorbit angle $\lambda$ is measurable via the Rossiter-McLaughlin effect. In addition, the stellar inclination $i_{\mathrm{s}}$ can in principle be constrained using asteroseismology. Nevertheless, we should keep in mind that the only angle of intrinsic physical significance is the spinorbit angle $\psi$ between $\mathbf{n}_{\mathrm{o}}$ and $\mathbf{n}_{\mathrm{s}}$ (not depicted in Fig. 1), which is generally not directly measurable. 

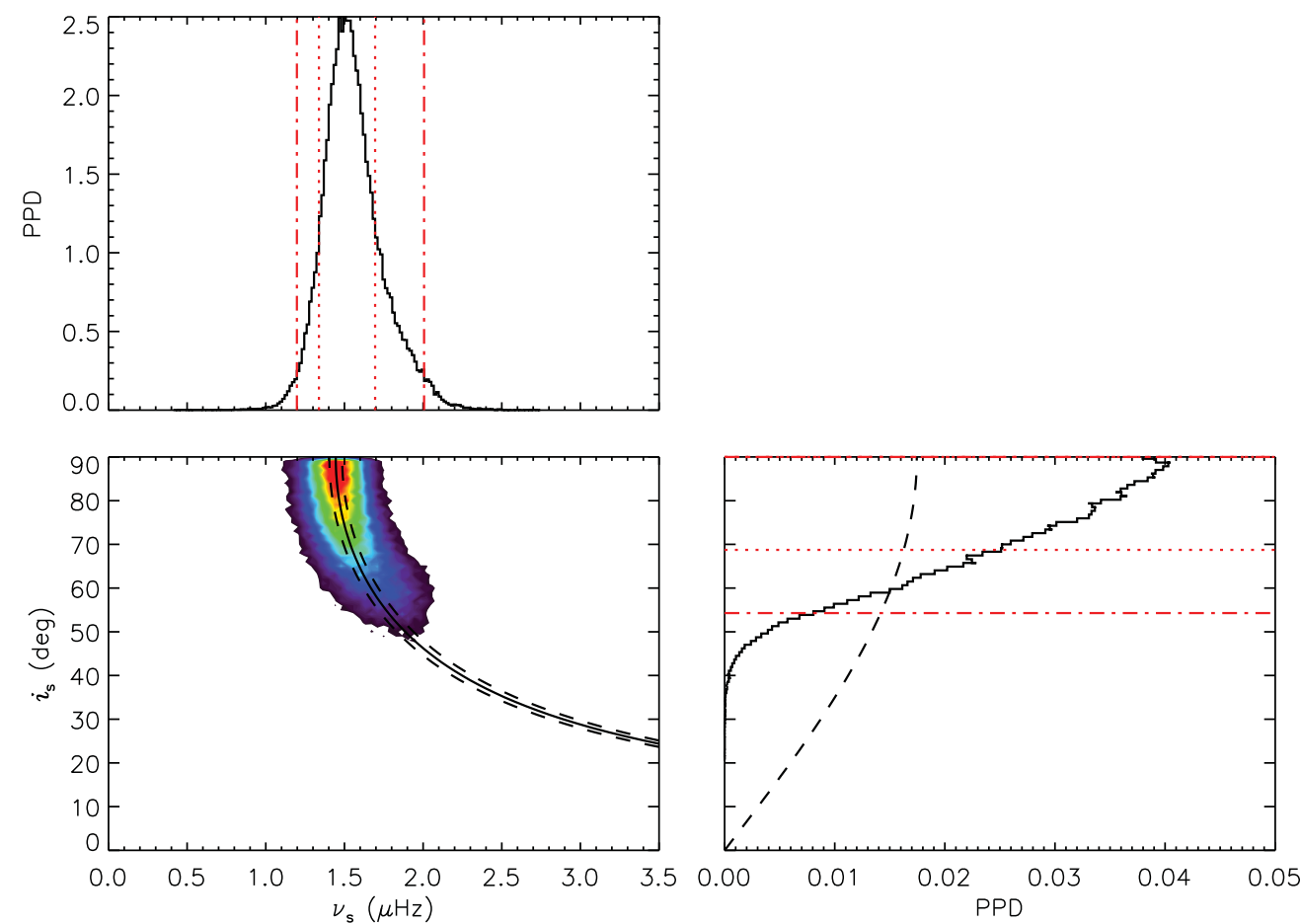

Figure 2. Asteroseismic results on KIC 4349452 (Kepler-25, KOI-244). Bottom left panel: Joint posterior probability distribution (PPD) of the stellar inclination, $i_{\mathrm{s}}$, and the rotational splitting, $\nu_{\mathrm{s}}$. Also shown is an estimate of the reduced splitting (solid line) with its associated 1- $\sigma$ envelope (dashed lines). Top left and bottom right panels: Marginalized PPDs. Dotted and dot-dashed lines respectively enclose the $68.3 \%$ and $95.4 \%$ credible regions. For reference, the dashed curve in the bottom right panel represents the (uninformative) isotropic prior on $i_{\mathrm{s}}$ adopted in the asteroseismic analysis.

\section{Asteroseismic analysis}

We have selected for analysis an asteroseismic sample of 25 solar-type candidate hosts (also designated as Kepler Objects of Interest or KOIs), of which 20 have been confirmed as planetary-system hosts. These range in spectral type from late F- to early K-type stars. There are 14 multi- and 11 single-transiting systems in our sample, with a clear prevalence of systems that contain small planets (i.e., $R_{\mathrm{p}}<4 R_{\oplus}$ ) and long-period planets (i.e., $P_{\mathrm{o}}>10 \mathrm{~d}$ ). Light curves optimized for asteroseismic analyses (Handberg \& Lund 2014) were generated for each of the sample stars and their respective power spectra fitted using a Markov chain Monte Carlo algorithm (MCMC; Handberg \& Campante 2011) that employed uninformative priors.

We look in detail at the case of the the multi-transiting system Kepler-25. Shown in Fig. 2 is a correlation map of the stellar inclination and rotational splitting, as obtained from the MCMC analysis. The posterior distribution of the stellar inclination is concentrated toward $90^{\circ}$ and is thus consistent with alignment. A measurement of the projected spin-orbit angle from the Rossiter-McLaughlin effect is also available for this system (Albrecht et al. 2013). By combining that information with the asteroseismic stellar inclination, we can constrain the true obliquity of the system (see Fig. 3). We find that Kepler-25c (the largest of the transiting planets in the system) is well aligned with 


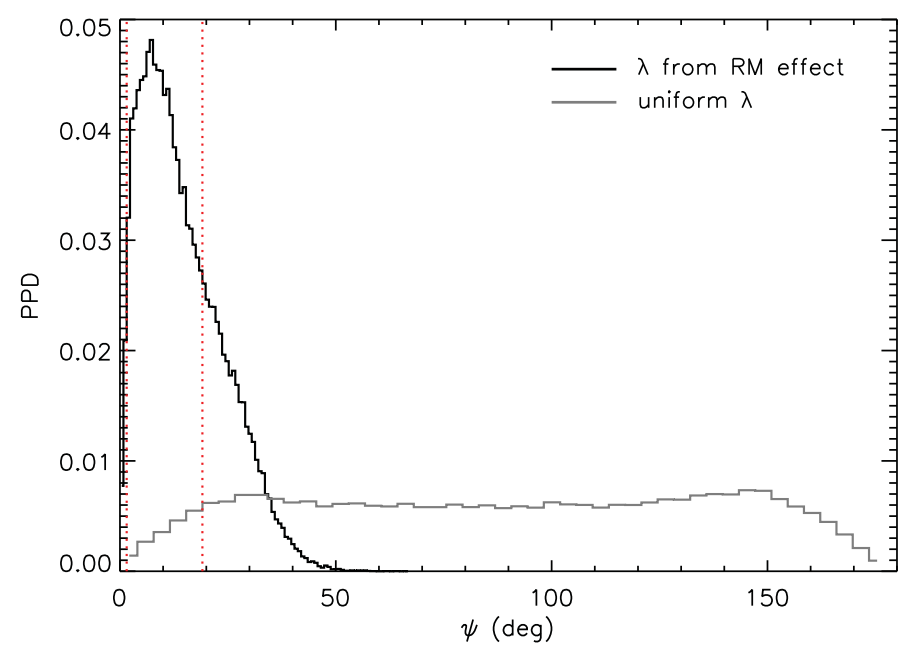

Figure 3. Posterior probability distribution of the spin-orbit angle $\psi$ of KIC 4349452 (Kepler-25, KOI-244). The black histogram was obtained by means of Monte Carlo simulations using the PPD of $i_{\mathrm{s}}$ from our analysis, and assuming normal and uniform distributions, respectively, for $\lambda$ and $i_{\mathrm{o}}$ around their adopted literature values. Dotted lines enclose its associated $68.3 \%$ credible region. The gray histogram was obtained by instead assuming an isotropic distribution in $\lambda$.

the stellar spin axis (with $\psi$ being close to $0^{\circ}$ ). Our findings are thus not in agreement with the statement made by Benomar et al. (2014) that this system is mildly misaligned.

\section{Statistical constraints on spin-orbit alignment}

Shown in Fig. 4 is the average posterior of the cosine of the stellar inclination, made by averaging over the individual posteriors. This is shown for all the 25 stars in the sample, as well as for the subsamples of single- and multi-transiting systems. As can be seen, the KOIs in the asteroseismic sample preferentially display large values of the stellar inclination, this being more accentuated than if these angles had been drawn from an isotropic distribution (which is represented by a dashed line). Furthermore, we find no significant difference between the posterior distributions of single- and multi-transiting systems.

\section{Implications}

The implications of this work can be easily summarized:

- Our results for the stellar inclination are consistent with alignment at the $2-\sigma$ level for all KOIs in the asteroseismic sample, meaning that the system surrounding the redgiant star Kepler-56 remains as the only unambiguous misaligned multiple-planet system detected to date.

- The ensemble analysis suggests that the directions of the stellar spin and planetary orbital axes are correlated, as conveyed by a tendency of the KOIs to display large values of the stellar inclination.

- This tendency is also true for multi-transiting systems. Under the assumption that the planetary orbits in multi-transiting systems are nearly coplanar and trace the plane of the protoplanetary disk, this would imply that the high obliquities observed for hotJupiter systems are associated with planet migration. Migration mechanisms capable 


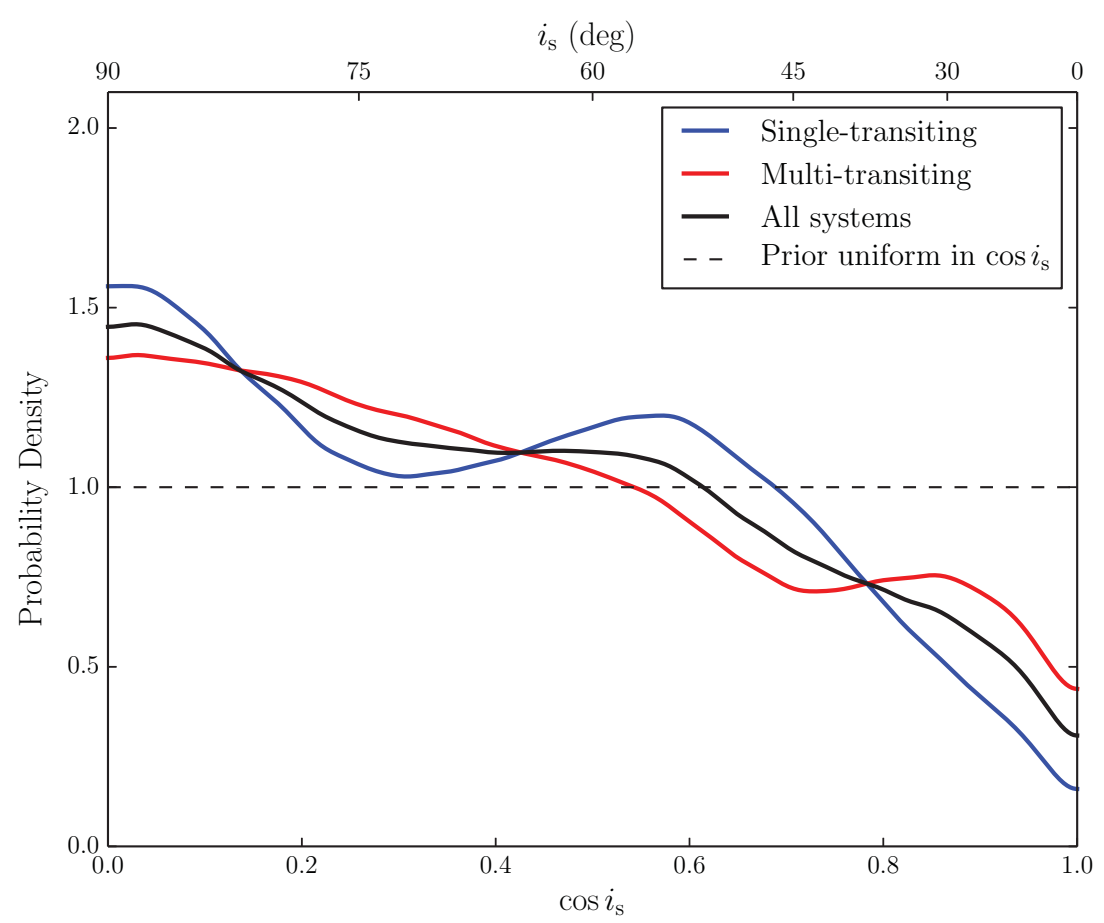

Figure 4. Average posterior probability distribution of $\cos i_{\mathrm{s}}$ (kernel density estimate) for stars in the asteroseismic sample. All systems have been assigned the same weight. The overall average posterior is depicted in black, whereas the average posteriors for single- and multi-transiting systems are depicted in blue and red, respectively. For reference, the horizontal dashed line represents the (uninformative) isotropic prior on $i_{\mathrm{s}}$ (or, equivalently, the uniform prior on $\cos i_{\mathrm{s}}$ ) adopted in the asteroseismic analysis.

of exciting high obliquities are thus favored over disk migration to explain hot-Jupiter formation.

- The constraints on spin-orbit alignment made possible by an analysis of the asteroseismic sample are in qualitative agreement with the outcome of the analysis presented in Hirano et al. (2014), who have estimated $i_{\mathrm{s}}$ for a sample of 25 (coincidentally) KOIs based on measurements of their rotation period, rotational line broadening, and stellar radius. Furthermore, our results do not support the recent finding by Morton \& Winn (2014) that the obliquities of systems with a single transiting planet are systematically larger than those with multiple transiting planets. We should stress, however, that the small size of our sample prevents us from drawing definitive conclusions.

\section{Outlook}

This work presents the first analysis of an ensemble of asteroseismic obliquity measurements made for transiting systems. The prospect of using asteroseismology to measure the obliquities of systems with evolved hosts will be addressed when data from the TESS mission (Transiting Exoplanet Survey Satellite; Ricker et al. 2015) become available. The planned PLATO mission (PLAnetary Transits and Oscillations of stars; Rauer et al. 2014) will further offer the possibility of extending these asteroseismic measurements to bright solar-type hosts in wide fields. 


\section{References}

Albrecht, S., Winn, J. N., Johnson, J. A., et al. 2012, ApJ, 757, 18

Albrecht, S., Winn, J. N., Marcy, G. W., et al. 2013, ApJ, 771, 11

Benomar, O., Masuda, K., Shibahashi, H., \& Suto, Y. 2014, PASJ, 66, 94

Chaplin, W. J., Sanchis-Ojeda, R., Campante, T. L., et al. 2013, ApJ, 766, 101

Gizon, L. \& Solanki, S. K. 2003, ApJ, 589, 1009

Gizon, L., Ballot, J., Michel, E., et al. 2013, Proc. of the National Academy of Science, 110, 13267

Handberg, R. \& Campante, T. L. 2011, A\&A A, 527, A56

Handberg, R. \& Lund, M. N. 2014, MNRAS, 445, 2698

Hirano, T., Sanchis-Ojeda, R., Takeda, Y., et al. 2014, ApJ, 783, 9

Huber, D., Carter, J. A., Barbieri, M., et al. 2013, Science, 342, 331

Lund, M. N., Lundkvist, M., Silva Aguirre, V., et al. 2014, A\&A, 570, A54

Morton, T. D. \& Winn, J. N. 2014, ApJ, 796, 47

Rauer, H., Catala, C., Aerts, C., et al. 2014, Experimental Astronomy, 38, 249

Ricker, G. R., Winn, J. N., Vanderspek, R., et al. 2015, Journal of Astronomical Telescopes, Instruments, and Systems, 1, 014003

Wright, D. J., Chené, A.-N., De Cat, P., et al. 2011, ApJ (Letters), 728, L20 